\title{
A Decision Model and Support System for the Optimal Design of Health Information Networks
}

\author{
Oded Berman, Fatemeh "Mariam" Zahedi, and Kim R. Pemble
}

\begin{abstract}
Health information networks (HINs) have become increasingly important in the structure of the health care industry. In this paper, we develop decision models and decision-support systems for designing and performing cost-benefit analysis of HINs. Our models prioritize the connection of various types of health providers to the network based on the costs and benefits of all participants: the network owners, information providers, and information users of the HIN. The business strategy underlying this analysis is to design the system with the maximum value for the network owner(s), while ensuring that the network would provide positive added-value to each of its nodes. Our framework could be used to examine the design and perform cost and benefit analysis for an existing network, the expansion of an existing network, or the development of a new network. One can also use the models for break-even point and scenario analysis. We have used our approach to examine an existing HIN [Wisconsin Health Information Network (WHIN)] and to perform scenario analysis for a possible restructuring of the network. Our empirical results in this case show that HINs could be highly profitable for all network participants.
\end{abstract}

Index Terms-Community health information networks (CHINs), health information networks (HINs), optimization, scenario analysis, Wisconsin Health Information Network (WHIN).

\section{HEALTh INFORMATION NETWORKS (HINs)}

$\mathbf{H}$ EALTH care has sbecome one of the most important service industries, and is undergoing rapid structural transformations [48]. A number of factors in these transformations have made access to health information and consequently information networks critical success factors for health organizations. One driving factor in this process is the expansion of for-profit managed care, which has fueled the consolidation of health providers and has created geographically diverse health conglomerates in need of networking [49]. "Hospitals, physicians, insurers, and managed-care firms are networking, merging, and forming horizontally and vertically integrated organizations to finance and deliver health care" [8].

A number of factors are driving the emerging prominence of health information networks (HINs). Health industry participants, in various forms, have begun to leverage the technology trends for organizational change [1], [23], [38]. The second

\footnotetext{
Manuscript received December 9, 1999; revised May 10, 2001. This paper was recommended by Associate Editor R. Rada.

O. Berman is with the Joseph L. Rotman School of Management, University of Toronto, Toronto, ON, M5S 3E6 Canada (e-mail: berman@ fmgmt.mgmt. utoronto.ca).

F. Zahedi is with the School of Business, University of Wisconsin, Milwaukee, WI 53201 USA (e-mail: zahedi@uwm.edu).

K. R. Pemble is with the School of Allied Health Professions, University of

Wisconsin, Milwaukee, WI 53201 USA (e-mail: krpemble@sahp.uwm.edu).

Publisher Item Identifier S 1094-6977(01)06488-4.
}

factor is the movement of the patient from one provider to another within the same managed-care system, which has heightened the need for accurate and timely exchange of patient-focused, integrated information at multiple sites and across providers [1], [11], [12], [17]. The third factor is physicians' growing dependency on managed plans and hospitals as they earn a smaller portion of their income from fee-for-service care, increasing their need to access patients' records from hospitals and testing facilities on the fly. In one 1995 survey, $87 \%$ of practicing physicians participated at least in one managed care plan [48]. The fourth factor is the providers' need to connect with insurers for eligibility authorization, referrals, pharmacy formularies, billing, and at times to larger employers, who directly buy health services for their employees. The fifth factor is the emergence of telemedicine and its dependency on information networks [10], [18], [28], [42], [43]. The sixth factor is large health providers' view of technology as a means of providing specialized, cost-effective care for patients in remote and rural areas [21], [24], [44], [47], [50], [52]. The successful use of networking technology in other industries has prompted the health industry to look into health networks as one important approach in dealing with these complex factors.

A market survey reported that there are more than $500 \mathrm{HINs}$ in the United States that have major activity in communities [20]. In the annual survey of health-care executives, $70 \%$ reported that they have either completed or are actively working on a system that makes patient information and histories available throughout the organization [35]. The biggest push is to connect physicians' offices with hospital information systems [34]-[37].

Brennan [4, p. 2] defines an HIN as: "an integrated collection of computer and telecommunications capabilities that facilitate the exchange of patient, clinical, and financial information among physicians, hospitals, payers, employers, pharmacies, and related health care entities within a geographical region." HINs could have different structural forms: enterprise-owned (including vendor-owned) and open system (including community- or consortium-owned).

An enterprise-owned HIN is designed to connect various units, providers, services, and participants within a given enterprise. Such HINs normally have a centralized structure. Examples of such a structure are Covenant Health Care in Wisconsin, its parent company, the Wheaton Franciscan (which operates similar systems in Iowa and other states), and Project Spectrum in Missouri and southern Illinois [25].

The open system concept of health information systems is the community health information network (CHIN). The advantage 
of such a system is the "community" concept, with its interorganizational exchange of information among health-care entities [15], [39]. The CHINs idea began with creating a centralized MIS system for community health, in which a central repository would aggregate and store data and make the data available to a broad spectrum of community users [46]. However, creating a central database at the community level raises serious issues of data ownership and confidentiality [45]. The centralized approach is limited to the enterprise-level or government-level HINs that could manage issues related to data ownership and confidentiality. The decentralized approach to information exchange in CHINs is more acceptable to health industry participants and creates fewer problems regarding confidentiality and ownership of patient data [46]. Hence, many CHIN initiatives use a decentralized approach in their system design. The CHIN in the greater Dayton area has been implemented through the partnership of area hospitals, Ameritech, IBM, and the University of Dayton for sharing patient medical information among independent hospitals [19]. There are projects, some at the pilot stage, for using the Internet as the telecommunication backbone for creating CHINs with limited or full functionalities [15], [27], [22].

There are both public-sector and private-sector ownership models for CHINs. An example of public-sector initiative at the federal level is the Centers for Disease Control and Prevention's creation of an integrated infrastructure for the Information Network for Public Health Officials [2], [9]. An example of the state initiative is the investment by the state of Iowa in building a fiber-optic network to link 99 county seats, enabling the University of Iowa to set up a rural telemedicine experiment, called the National Laboratory for the Study of Rural Telemedicine. This laboratory uses the information systems of the university's hospitals and clinics to provide telemedicine to rural areas in Iowa.

The private sector has shown the most initiative in creating CHINs. The alliance of telecommunication companies and health care providers in creating HINs and CHINs is a common occurrence. Pacific Bell has developed the Bell Health Information Network, which "is expected to be used by half of California's health care providers covering a total of 11 million lives" [6]. In 1995, Ameritech Corporation (Chicago, IL) announced that Blue Cross Blue Shield of Illinois had hired it to provide an information network for 6300 members of the Blues' managed-care physicians group [6]. In 1992, a joint venture of Aurora Health Care and Ameritech created the Wisconsin Health Information Network (WHIN) [53].

Single ownership of CHINs may prove to be unsuccessful. Ameritech established the Regional Health Information Network of Northern Ohio (RHINNO) in 1994, but it encountered resistance from the business and medical communities [18]. The popular sentiment is toward CHIN's ownership by consortia of hospitals, physicians, payers, and business interests, as shown in a survey [34].

In their study of CHINs, Payton and Brennan [41] categorize CHINs in groups including telephone-accessible (including Internet-based), voluntary, and enterprise networks. The telephone-based and Internet-based CHINs have emerged as low-cost alternatives to deliver medical information and self-care services to patients through the Internet, voice mail, and conference calls. These networks are not motivated by financial gains and supported by nonprofit organizations, including local governments, universities, and community agencies [41]. On the other hand, voluntary networks parallel the decentralized for profit networks, whereas the enterprise-wide networks are mostly design to integrate constituent systems to deliver an integrated delivery approach, which has a more centralized and intraorganizational focus. In this paper, our focus is primarily voluntary networks, although our results could be used by enterprise-wide networks, as well.

The creation of an HIN and the participation in it require serious commitments by all entities involved. However, there is no formal mechanism for guiding the diverse and often divergent groups of clinicians, health managers, and technologists through the maze of analyzing and designing such networks. Delesie [13] has already observed the potentially critical role of academic research in bridging such gaps in the health care industry, and is demonstrated by Bretthauer and Côté [7] in modeling resource requirements in health care organizations. Furthermore, in their review of 333 publications on the management of health care technology, Kumar et al. [29] have identified cost performance to be the most pursued objective in the field.

In this paper, we develop a framework to identify the design of the network and to carry out scenario analysis for the costs and benefits of network designs. The application of our framework in analyzing WHIN shows the usefulness of our approach. Our empirical analysis has led us to conclude that health information systems could be quite profitable for all participants.

This paper has the following organization. Section II defines the problem. Section III formulates the decision models, and reports on the structure of the decision support system for using the models. Section IV analyzes the application of the models to the WHIN. Section V redesigns the WHIN architecture and provides the decision model to accomplish it. Section VI offers concluding remarks.

\section{PROBlem Definition}

An HIN can be viewed as a network of interconnected nodes. An information request originates from a node and is sent to another node in the network. A node can be an information provider or information user [40]. In WHIN at present, for example, a node is either a provider of information or a user of information but not both [53]. (There are exceptional cases, where a node could be set up to act as both the provider and user of information in WHIN.)

As a network expands and the extent of connectivity increases, a node becomes both the provider as well as the user of information. For example, a physician's office needs to access patients' hospital records, and to send its office records to labs or emergency rooms at various hospitals. This makes the physician's office both user and provider of information. If the physician is allowed to admit the patient to the hospital via the 
network from his/her office, then the physician's office not only provides the information to the hospital for use, it also provides the information for record updates. Therefore, as the scope of data exchange in the network increases, the interactivities among nodes increase, and the boundaries between the provider and user of information disappear.

We define network nodes as those units connected to the network, providing and/or using information. There are cost and benefit consequences in adding nodes to the network. As a node is added to the network, it provides information to other nodes; hence it generates benefits to all nodes that are connected to the network. However, nodes will not equally benefit from the information provided to the network. For example, if a hospital is added to the network, physicians and clinics that have dealings with that hospital will benefit most from the added node. Other hospitals that are located far away from the added node and have infrequent dealings with it will not benefit substantially from the addition of the node. Hence, the addition of a node creates a benefit for the entire network. However, the existing nodes would not have an equal share in the added benefit.

Another consequence of adding a new node is the benefit it receives from this connectivity. The benefit of joining a network could be tangible, such as saving cost or manpower, or intangible, such as increased physician satisfaction, faster service, or higher quality of care.

A third benefit is the ability of the network owner to sell network time to the node and make a profit from it. For example, a CHIN in the Midwest charges a fixed rate of $\$ 75$ per month for connecting 15 physicians in a clinic and $\$ 0.50$ per query per patient. In other cases, the node may also contribute to the equity ownership of the network and share the cost of setting up the network infrastructure and its maintenance.

Adding a new node also involves costs to the node and the network. The cost for the node could be divided into two parts: internal and external. The node's internal cost is what it has to invest in order to make its connectivity possible. For example, when a physician's office connects to a CHIN, it has to have the right computers, software, communication lines, and staff to handle the electronic exchange of information. The external cost for the node is its payment to the network.

The cost to the network is providing the needed services and possible interfaces to make the connection possible. Furthermore, as new nodes are added and the amount of information exchange increases, the speed of exchange may decrease and the network owner will need to expand the capacity of the network or even upgrade it to a more complex system.

Hence, designing a network or prioritizing the addition of nodes to a network involves a complex set of decision variables. The question that we address here is to provide a decision framework for designing a network configuration that optimizes the total value of the network, and provides a framework for a rigorous cost-benefit analysis in the design of the network.

\section{DECISION MODELS}

In developing a decision model for network design, the focus of analysis impacts the formulation. One focus could be the de- velopment of a new network. A slightly different focus is the break-even point analysis for answering the question of how many nodes should be connected to a network to make it viable. Yet, a third decision focus may be the analysis of new additional nodes to an existing network. A fourth case is when nodes are numerous and could be categorized into groups of homogeneous nodes. This last case in turn could have one of the above three focuses. In our empirical work, we began with the first focus in our modeling process and, by answering questions raised at different stages of our study, ended with the fourth one. We present all four models here, and report the application of the fourth case in Sections IV and V.

We formulate the problem from the for-profit network owner's point of view. The owner could be a single entity or a consortium. The business strategy guiding this formulation is that the owner maximizes its total profit, while ensuring positive added value to the network participants. Furthermore, we assume the following.

- Node $i$ will want to be connected only if the charge is less than or equal to the total benefit it receives.

- Benefit to node $i$ is the sum of all the information values provided by the other connected nodes.

- Benefits are translated to monetary values.

Notations (Costs, Benefits, and Values are in Monetary Terms): $n \quad$ Potential number of nodes.

$v_{i j} \quad$ Value of information added to node $i$ by node $j$.

$b_{i} \quad$ Benefit of connection to the network for node $i$.

$c_{i} \quad$ Cost for adding node $i$.

$p_{i} \quad$ Charge for connecting node $i$ to the network (takes into account estimated usage of the network).

$X_{i} \quad$ One if node $i$ is to be connected to the network, zero otherwise.

$O\left(\sum_{i=1}^{n} X_{i}\right) \quad$ Overhead cost-a step function of number and identity of customers connected.

$Y_{i} \quad$ One if node $i$ is interested in being connected, given charge $p_{i}$.

$Z_{i} \quad$ Binary variable.

$U \quad$ Very large number.

$F_{i} \quad$ Overhead start-up cost for level $i$, where a level represents a given number of nodes.

\section{A. Decision Model for Connectivity in a New Network (D1)}

The purpose of this model is to aid in deciding which candidate nodes should be connected to the network. The objective is to maximize the profit of the network owners, ensuring that user nodes receive at least as much value as they pay to the network owner.

Following the second assumption, the total benefit to node $i$ is computed as

$$
b_{i}=\sum_{j=1}^{n} v_{i j} X_{j} .
$$

Node $i$ will desire to be connected to the network $\left(Y_{i}=1\right)$ if the benefit it receives is higher than the price it pays, hence: $b_{i} \geq p_{i}$. Otherwise, node $i$ will not be connected and $Y_{i}=0$. 
Therefore, the model could be formulated as a linear integer optimization as

D1)

$$
\begin{array}{ll}
\max & \sum_{i=1}^{n} p_{i} X_{i}-\sum_{i=1}^{n} c_{i} X_{i}-O\left(\sum_{i=1}^{n} X_{i}\right) \\
\text { s.t. } & \\
& X_{i} \leq Y_{i} \quad i=1,2, \ldots, n \\
& \sum_{j=1}^{n} v_{i j} X_{j} \geq p_{i} Y_{i} \quad i=1,2, \ldots, n \\
& X_{i}, Y_{i}=0,1 \quad i=1,2, \ldots, n .
\end{array}
$$

The objective function maximizes the total profit. The first term consists of fees paid to the network owner as revenue and the second term is the network owner's variable cost. The third term is the fixed overhead (start-up) cost of the network that depends on the number and identity of nodes in the network. The third term will be reformulated below. Equations (2) and (3) guarantee that node $i$ can be connected only if node $i$ wishes to be, and node $i$ will wish to be connected only if the total benefit it receives is equal to or larger than the fees it pays.

The third term in the objective function, $O\left(\sum_{i=1}^{n} X_{i}\right)$, is a step function with $m$ levels (excluding the zero level), and can be presented as follows:

$$
\begin{aligned}
& O\left(\sum_{i=1}^{n} X_{i}\right)=O_{0}=0, \quad \text { if } \sum_{i=1}^{n} X_{i}=a_{0}=0 \\
& O\left(\sum_{i=1}^{n} X_{i}\right)=O_{1}+\sum_{i=1}^{n} F_{i} X_{i}, \quad \text { if } \sum_{i=1}^{n} X_{i} \leq a_{1} \\
& O\left(\sum_{i=1}^{n} X_{i}\right)=O_{k}+\sum_{i=1}^{n} F_{i} X_{i}, \quad \text { if } \sum_{i=1}^{n} X_{i} \leq a_{k} \\
& O\left(\sum_{i=1}^{n} X_{i}\right)=O_{m}+\sum_{i=1}^{n} F_{i} X_{i}, \quad \text { if } \sum_{i=1}^{n} X_{i}>a_{m-1} .
\end{aligned}
$$

The levels of the overhead model show a network of variable size. Therefore, as the number of nodes connected to the network increases, the capacity of the network is exhausted and new investment is needed to expand its capacity. Hence, the fixed overhead jumps from one level to the next.

Assuming that $O\left(\sum_{i=1}^{n} X_{i}\right)$ is a nondecreasing cost function, we can insert it into our formulation and have a pure integer programming problem as follows. We replace $O\left(\sum_{i=1}^{n} X_{i}\right)$ in the objective function by

$$
\sum_{k=0}^{m} O_{k} Z_{k}+\sum_{i=1}^{n} F_{i} X_{i}
$$

and introduce additional constraints as

$$
\begin{aligned}
\sum_{k=0}^{m} Z_{k} & =1 \\
\sum_{i=1}^{n} X_{i} & \leq \sum_{k=0}^{m-1}\left(a_{k}+0.5\right) Z_{k}+U Z_{m} \\
Z_{k} & =0,1 \quad k=0,1, \ldots, m .
\end{aligned}
$$

Solving the D1 model (1)-(8) gives us the optimum design for the network configuration.

\section{B. Decision Model for Break-Even Analysis of Nodes (D2)}

In designing a network from scratch, one needs to know the minimum number of nodes that must be connected for the network owner to reach a break-even point. This is important when the network is being designed for the first time and the network owner needs to identify the nodes, which should receive the highest priority as its founding customers. This means that we should find the minimum $n$ and the identity of the nodes that will make the network viable. This can be achieved by solving the following optimization problem:

D2)

$$
\begin{array}{ll}
\min & \sum_{i=1}^{n} X_{i} \\
\text { s.t. } & \\
& \sum_{i=1}^{n} p_{i} X_{i}-\sum_{i=1}^{n} c_{i} X_{i}-O\left(\sum_{i=1}^{n} X_{i}\right) \geq 0.01 \\
& X_{i} \leq Y_{i} \quad i=1,2, \ldots, n \\
& \sum_{j=1}^{n} v_{i j} X_{j} \geq p_{i} Y_{i} \quad i=1,2, \ldots, n \\
& X_{i}, Y_{i}=0,1 \quad i=1,2, \ldots, n .
\end{array}
$$

The formulation in this problem is similar to that in D1, with the exception that the objective function of the model (9) is the minimum number of nodes in the system, and the total profit of the network owner enters as a new constraint (10). This constraint is set slightly above 0 (e.g., 0.01 ) to exclude zero nodes as an acceptable solution.

\section{Decision Model for Adding New Nodes (D3)}

Another decision in designing a network is to add a new set of nodes to an existing network. There may be several potential new nodes. The question is which ones should be added to the network. This guides the network owner about the nature of the new customers it has to seek to expand the network.

To formulate this model, let $N_{1}$ (with power $n_{1}$ ) and $N_{2}$ be, respectively, the set of existing and potential nodes. Suppose that there is a decision to add a maximum of $h$ nodes to the network ( $h$ could be equal to 1 ; in which case we consider adding one new node). Then the decision model can be formulated as

D3)

$$
\begin{array}{ll}
\max & \sum_{i \in N_{2}} p_{i} X_{i}-\sum_{i \in N_{2}} c_{i} X_{i}-O\left(n_{1}+\sum_{i \in N_{2}} X_{i}\right) \\
+ & \sum_{i \in N_{1}} \sum_{j \in N_{2}} X_{j} v_{i j} \\
\text { s.t. } & \\
& \sum_{i \leq Y_{i}} v_{i j} X_{j}+\sum_{j \in N_{1}} v_{i j} \geq p_{i} Y_{i} \quad i \in N_{2} \\
& \sum_{j \in N_{2}} X_{j \in N_{2}} X_{i} \leq h
\end{array}
$$


where

$$
\begin{aligned}
& O\left(\sum_{i \in N_{2}} X_{i}+n_{1}\right) \\
& =O_{0}=0 \text { if } \sum_{i \in N_{2}} X_{i}+n_{1}=a_{0}=0 \\
& O\left(\sum_{i \in N_{2}} X_{i}+n_{1}\right) \\
& \quad=O_{1}+\sum_{i \in N_{2}} F_{i} X_{i} \text { if } \sum_{i \in N_{2}} X_{i}+n_{1} \leq a_{1} \\
& O\left(\sum_{i \in N_{2}} X_{i}+n_{1}\right) \\
& \quad=O_{k}+\sum_{i \in N_{2}} F_{i} X_{i} \text { if } \sum_{i \in N_{2}} X_{i}+n_{1} \leq a_{k} \\
& O\left(\sum_{i \in N_{2}} X_{i}+n_{1}\right) \\
& \quad=O_{m}+\sum_{i \in N_{2}} F_{i} X_{i} \text { if } \sum_{i \in N_{2}} X_{i}+n_{1}>a_{m-1}
\end{aligned}
$$

Assuming that $O\left(\sum_{i \in N_{2}} X_{i}+n_{1}\right)$ is a nondecreasing cost function, we can insert it into our formulation and have a pure IP problem as follows. We replace $O\left(\sum_{i \in N_{2}} X_{i}+n_{1}\right)$ in the objective function by

$$
\sum_{k=1}^{m} O_{k} Z_{k}+\sum_{i \in N_{2}} F_{i} X_{i}
$$

This formulation differs from that in (5) because the existing nodes $\left(n_{i} \mathrm{~s}\right)$ enter in the step functions as fixed parameters. We also need to introduce additional constraints

$$
\begin{aligned}
\sum_{k=0}^{m} Z_{k} & =1 \\
\sum_{i \in N_{2}} X_{i}+n_{1} & \leq \sum_{k=0}^{m-1}\left(a_{k}+0.5\right) Z_{k}+U Z_{m} \\
Z_{k} & =0,1 \quad k=0,1, \ldots, m .
\end{aligned}
$$

The decision model D3 (14)-(22) determines which of the $h$ new potential nodes should be added to the network.

\section{Decision Model for Analyzing Categories of Nodes (D4)}

In the process of our empirical investigation of using D1-D3 models, it became evident that in a large network, data requirements for individual nodes may become prohibitively expensive. In many cases, homogeneous nodes could be grouped into one category with the same or similar values for parameters. Most importantly, analyzing categories of nodes would be more informative for cost-benefit analysis than individual nodes in making decisions regarding the addition of a group of nodes and the related investment. For example, adding insurance compa- nies to the network could have a radical impact on the design and implementation of the network. Such an addition could not be easily analyzed when only one insurance company is considered.

Formulating the decision problem for categories of nodes requires data for node categories only, and the actual number of connected nodes in each category is left for the model to determine. We allow for the possibility of setting an upper bound on the number of potential nodes in each category as well as on the maximum total number of nodes in the network.

To accomplish this, we change model D1 to create D4 in the following manner. Constraints (2) and (4) in D1 are changed to (24) and (27) to accommodate categorization and constraint (26) is added in order to put a possible limit on the total number of nodes in the network. Hence, we have

D4)

$$
\begin{array}{ll}
\max & \sum_{i=1}^{n} p_{i} X_{i}-\sum_{i=1}^{n} c_{i} X_{i}-O\left(\sum_{i=1}^{n} X_{i}\right) \\
& \\
& X_{i} \leq M_{i} Y_{i} \quad i=1,2, \ldots, n \\
& \sum_{j=1}^{n} v_{i j} X_{j} \geq p_{i} Y_{i} \quad i=1,2, \ldots, n \\
& \sum_{i=1}^{n} X_{i} \leq M \\
& X_{i} \geq 0 \text { and integer, } Y_{i}=0,1 \quad i=1,2, \ldots, n .
\end{array}
$$

Here, $n$ is the number of node categories, $X_{i}$ is the number of nodes in category $i$, and $Y_{i}$ is the variable that is set equal to 1 if at least one node of category $i$ is selected. Constraint (24) ensures that the number of nodes in each category does not exceed $M_{i}$ - the maximum number of nodes in that category. The network's capacity could be constrained to the maximum of $M$ nodes. Note that if $Y_{i}=1$ holds, the constraint (25) holds for any of the $X_{i}$ nodes selected for category $i$. The parameters $p_{i}$, $c_{i}$, and $v_{i j}$ are defined to be the same for any node that belongs to category $i$.

The model D4 not only determines which categories should be included in the network, it also determines the number of nodes in each category. We can formulate models equivalent to D2 and D3 by making similar adjustments in their constraints.

\section{E. Decision Support Systems}

We have developed four decision support systems (DSS-1, DSS-2, DSS-3, and DSS-4), based on the above four models. The systems are programmed in Visual Basic for Applications in Excel. Each system allows the user to input the parameters in the Excel worksheet, asks the user for the number of nodes, levels of overhead, and the related overhead values. It then automatically sets up the model, solves it using Excel Solver, and provides the user with the optimal solution. An example of the user interface is given in Fig. 1.

In the example shown in Fig. 2 for the D1 model, $n=5$, $m=2, U=440, a_{1}=0, a_{2}=2, O_{1}=0, O_{2}=8$, and 


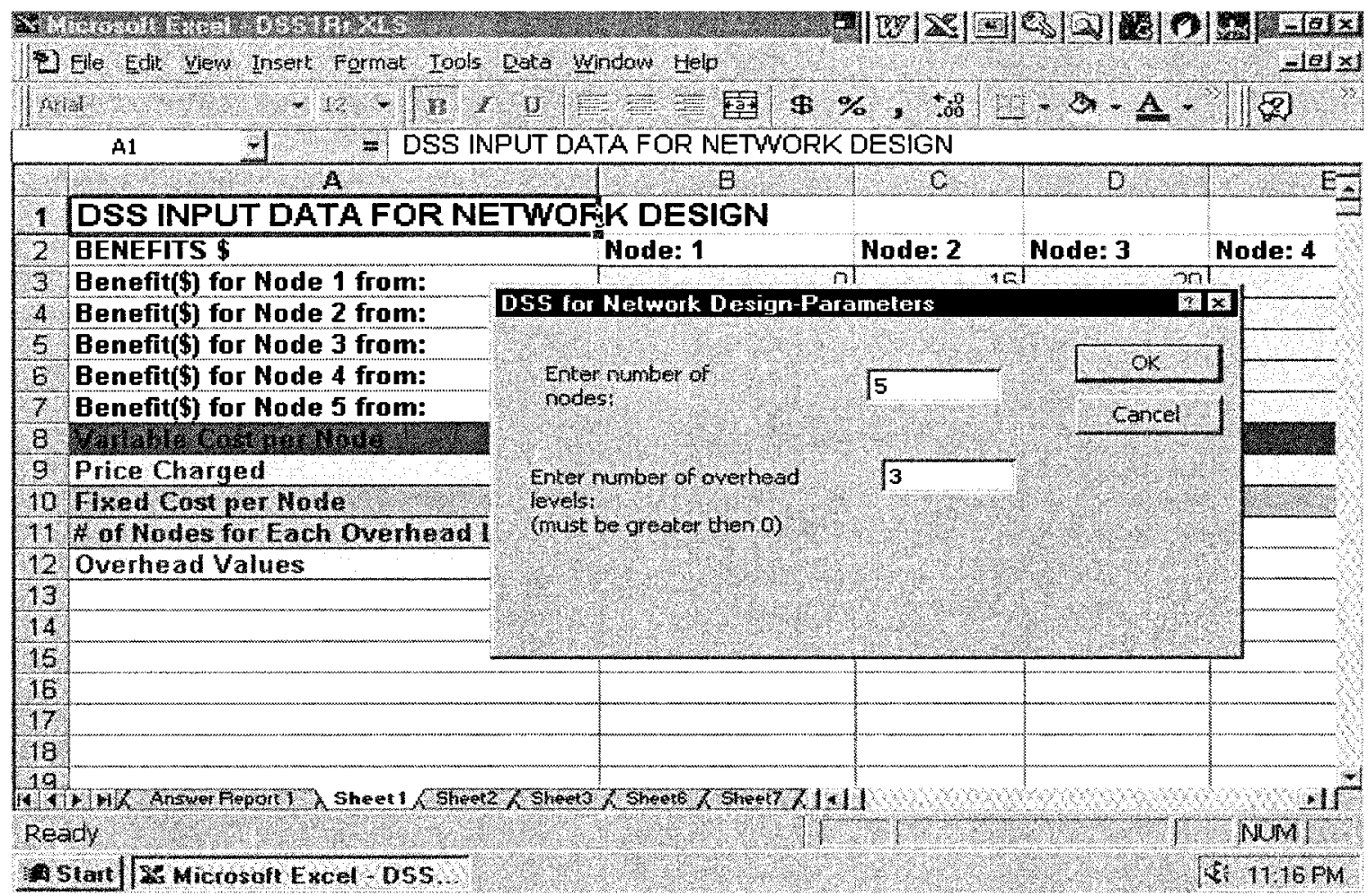

Fig. 1. Example of user interface for DSS-1.

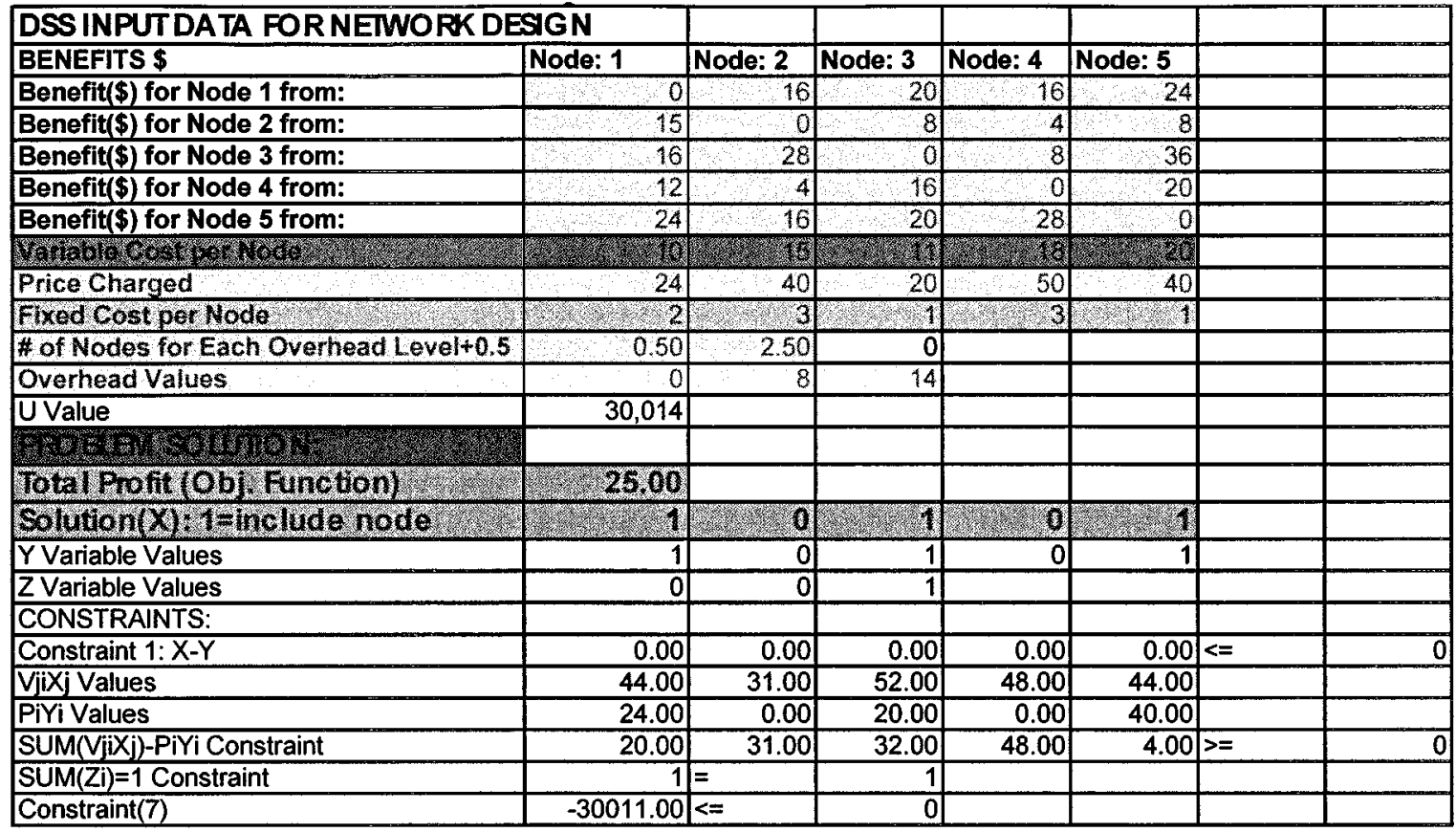

Fig. 2. Example in DSS-1 using model D1

$O_{3}=14$. The other parameters also are in the Excel worksheet. The optimal solution, shown in Fig. 2, is: $X_{1}=X_{3}=X_{5}=1$ with objective function value of 25 . Also, $Y_{1}=Y_{3}=Y_{5}=1$, $Z_{3}=1$. Obviously, this solution is intuitive. Since $\sum_{j=1}^{n} v_{2 j}=$ $35<p_{2}=40, X_{2}=0$. Suppose $X_{1}=X_{2}=X_{4}=X_{5}=1$. But then $\sum_{j=1}^{n} v_{4 j} X_{j}=48<p_{4}=50$ and thus we should have $X_{4}=0$ in the optimal solution. Solving this example in D2 - the break-even nodes-we find from DSS-2 that the number of nodes required to reach the break-even point is three. We then use this example for adding new nodes, assuming that existing nodes are $N_{1}=\{1,2,3\}$ (hence $n_{1}=3$ ) and new nodes are $N_{2}=\{4,5\}$. Using DSS- 3 gives us the optimal solution of $X_{4}=0, X_{5}=1$ with an optimal objective function of 73 . 


\section{ApPlication to the Wisconsin Health InFORMation NETWORK (WHIN) CASE}

In this section, we report on the analysis of the costs and benefits of the existing architecture of WHIN. In the next section, we examine changing the architecture to include retrieval of patient information not only from hospitals, but also from all nodes, thus treating each node simultaneously as both a provider and user of information.

Wisconsin is among the pioneering states in developing an HIN. The WHIN is a joint venture between Aurora Health Care and Ameritech that started in 1992 [40, p. 74]. At the start, these two companies owned the network, but made it open to ownership by other health care organizations in Wisconsin. In the summer of 1997, Ameritech sold its interest in WHIN to Aurora. Aurora continues to maintain the principle of open ownership by other organizations active in the health care industry.

Although the philosophy of HINs is sharing patient data among all nodes, in its present implementation, WHIN has two types of nodes: providers of information and users of information. Hospitals are the providers of information and other nodes, including hospital departments, are mainly information users. The network not only provides information exchange on medical records, it also offers the users a host of other services, such as claim submission, insurance eligibility verification, clinical reference systems (educational materials for patients), referrals, and e-mail. Some additional services include Internet access, Internet browser access for home and remote use, and outbound fax.

As Pemble [40] reports, WHIN connects 16 hospitals, eight clinics, seven insurance organizations, more than 1300 physicians, and eight ancillary organizations such as labs, pharmacies, transportation services, and home health agencies. However, membership in the network has been dynamic, with new connections and discontinuation of existing connections a common occurrence. At present, WHIN connects 14 hospitals, seven insurance companies, more than 1300 physicians, eight clinics, and 20 ancillary organizations, including ambulance services, private billing services, nursing homes, and home health agencies [53]. For our present study, we collected eight months of data on WHINs more than 220 nodes of information users with various levels of activity. (Note that a group of physicians may be one user node.)

Since the purpose of our study was to analyze the cost-benefit of categories of nodes for WHIN, we categorized the information user nodes into ten categories:

1) clinics (small, medium, and large);

2) MD offices (small, medium, and large);

3) billing services;

4) nursing services;

5) ambulance;

6) hospital departments.

The information provider hospitals were categorized into small, medium, and large.

Clinics and physicians' offices overlap in many ways, and are not distinguishable by their names. A physician's office may be much larger in the size and scope of work than another office that is called a "clinic." For consistency of analysis, we used the number of physicians as the criterion for distinguishing between clinics and MD offices, as well as in categorizing them by size. This criterion was used in analyzing the WHIN data.

We defined an office with four or fewer physicians as an MD office, and an office with five or more physicians as a clinic. A small MD office has one physician, a medium MD office has two or three physicians, and a large MD office has four physicians. A small clinic has five to ten physicians, a medium clinic has 11 to 25 physicians, and a large clinic has more than 25 physicians. We used the WHIN data to arrive at these categories. We also categorized information-provider hospitals into small (average of 100 staff physicians and 100 beds), medium (average of 250 staff physicians and 250 beds), and large (average of 500 staff physicians and 500 beds).

Since the data for nodes within each category were similar, we were able to perform cost-benefit analysis using the D4 model. This model requires the estimation of parameters for fee charges ( $p_{i}$ values), costs ( $c_{i}$ and $f i$ values), and information values ( $v_{i j}$ values) for categories of nodes.

Network Owner Fee Charges and Costs: WHIN charges its nodes a one-time entry cost and a monthly fee. The fixed one-time entry fee covers the software, training, and start-up costs. The monthly fee is based on the type of information exchange (such as medical records, electronic claim submission, and eligibility verification) as well as the number of transactions in each category. (There is a second type in which a flat monthly fee replaces the variable transaction charges for medical records.)

The determining factors in the variable monthly fee structures are mainly the number of physicians, number of users, number of concurrent users (number of possible simultaneous connections to the network), number of workstations, and the existence of a local area network (LAN). We used WHIN's data to estimate an average profile for each information-user category. Table I shows a summary of profiles for each information-user category. The user profiles were needed for estimating the fee charges.

Another piece of information needed for estimating the monthly fee charges was the number of monthly transactions in each node category. Since WHIN offers a variety of services, we needed to estimate the monthly transactions in each category of services. The transaction categories include clinical inquiries, claims submission, payor inquiries, clinical reference systems (CRS), i.e., educational literature for patients, referral, and e-mail. We collected monthly transactions for eight months (the data available to us) in order to estimate the average number of monthly transactions for each service type by each information-user node type. Table II reports a summary of these estimates.

An interesting observation is that the number of transactions per physician in clinics and MD offices are quite variable, and some smaller offices have a larger number of monthly transactions than larger offices, which could be considered an indication of more efficient use of the network. This is particularly true for medium-size and large clinics, which have elected not to utilize the network services in processing claims and CRS. Hospital departments' utilization of the network services, considering the number of their staff physicians and users, is rel- 
TABLE I

Average ProfiLes of INFORMATION USER NODE TyPeS

\begin{tabular}{|c|c|c|c|c|c|}
\hline Type & \# of MDs & \# of Users & $\begin{array}{c}\# \text { of } \\
\text { Concurrent } \\
\text { Users }\end{array}$ & $\begin{array}{l}\text { \# of Work- } \\
\text { stations }\end{array}$ & LAN \\
\hline Small Clinic & 6.2 & 20.7 & 2.5 & 2.4 & $\mathrm{~N}(90 \%)^{(f)}$ \\
\hline Med. Clinic & 14.6 & 47.0 & 6.4 & 5.0 & $\overline{\mathrm{N}(73 \%)}$ \\
\hline Large Clinic ${ }^{(*)}$ & 70.0 & 115.0 & 11.0 & 11.0 & $\mathrm{~N}$ \\
\hline Small MD & 1.2 & 4.8 & 0.8 & 0.8 & $\mathrm{~N}\left(80^{\circ} \%\right)$ \\
\hline Med. MD & 2.8 & 9.2 & 1.3 & 0.8 & $\mathrm{~N}(73 \%)$ \\
\hline Large MD & 5.7 & 17.1 & 2.1 & 1.6 & $\mathrm{~N}(79 \%)$ \\
\hline Billing & - & 32.3 & 2.2 & 1.3 & $\mathrm{~N}(83 \%)$ \\
\hline Nursing & - & 35.5 & 7.3 & 5.8 & $\mathrm{~N}(100 \%)$ \\
\hline Ambulance & - & 6.7 & 1.3 & 1.3 & $\mathrm{~N}(100 \%)$ \\
\hline Hos. Depts. ${ }^{\left({ }^{*}\right)}$ & 45.0 & 59.0 & 11.0 & 11.0 & $\bar{N}$ \\
\hline
\end{tabular}

TABLE II

Estimated AVERAge Number of MONTHLY TRANSACTIONS

\begin{tabular}{l|r|r|r|r|r|r}
\hline \multicolumn{1}{c|}{ Type } & Clinical & Claims & Payor & CRS & Referrals & E-mail \\
\hline Small Clinic & 513.6 & $1,820.0$ & 273.2 & 14.0 & 122.5 & 60.4 \\
\hline Med. Clinic & $1,544.1$ & - & 217.4 & - & 515.0 & 15.0 \\
\hline Large Clinic ${ }^{(*)}$ & $1,966.0$ & - & 4,017 & - & - & - \\
\hline Small MD & 123.0 & 211.2 & 40.5 & - & 228.0 & 76.8 \\
\hline Med. MD & 287.9 & 241.3 & 58.5 & - & - & 159.9 \\
\hline Large MD & 521.3 & $1,934.0$ & 158.0 & - & - & 103.2 \\
\hline Billing & $1,342.0$ & $2,587.0$ & 213.0 & - & - & 65.0 \\
\hline Nursing & 343.3 & - & 19.0 & - & - & 406.0 \\
\hline Ambulance & $1,146.3$ & - & $1,046.5$ & - & - & - \\
\hline Hos. Depts. ${ }^{*}{ }^{*}$ & $1,000.0$ & - & 120.0 & - & - & 200.0 \\
\hline
\end{tabular}

$\left.{ }^{*}\right)$ Not an average, an estimate of a typical case

(-) No data in this category

atively low-perhaps because they have their own system for such services. In this case, these health organizations have great opportunities for significant cost savings through system integration.

We applied the fee structure of WHIN to estimations reported in Tables I and II to compute the one-time flat fee and variable monthly fees for each node category. We also used WHIN data to estimate overhead costs for each category of nodes. The details for fee and cost structures are not reported here for confidentiality reasons.

User Benefits: WHIN is one of the pioneering HINs that has studied the cost savings in connecting to the network as opposed to the traditional system of patient information exchange. WHIN's impact study, based on a study of 20 offices, reports that there is on average a saving of $\$ 5.10$ per transaction by the medical records departments in hospitals, $\$ 2.50$ per transaction for clinical information requests by physician offices, and $\$ 1.00$ per transaction for electronic referral requests [30], [31], [40]. This report, however, does not show the pairwise benefit of connectivity. In other words, a clinic may have all its benefit from connecting to one hospital, or the benefit may be spread over five different hospitals, in which the physicians in the clinic treat
TABLE III

COST AND BENEFIT OF INFORMATION USER NODES OVER FIVE YEARS

\begin{tabular}{l|c|c|c|c|c}
\hline Type & Cost & Benefit & $\begin{array}{c}\text { Net Benefit } \\
\mathbf{S}\end{array}$ & $\begin{array}{c}\text { Net Benefit } \\
\text { per } \\
\text { Physician }\end{array}$ & $\begin{array}{c}\text { Net Benefit } \\
\text { per User }\end{array}$ \\
\hline Small Clinic & $\$ 122,473$ & $\$ 212,280$ & $\$ 89,807$ & $\$ 14,485$ & $\$ 4,339$ \\
\hline Med. Clinic & 81,102 & 275,970 & 194,868 & 13,347 & 4,146 \\
\hline Large Clinic & 297,531 & 535,920 & 238,389 & 3,406 & 2,073 \\
\hline Small MD & 23,176 & 49,620 & 26,444 & 22,037 & 5,509 \\
\hline Med. MD & 27,185 & 66,000 & 38,815 & 13,863 & 4,219 \\
\hline Large MD & 119,625 & 206,760 & 87,135 & 15,287 & 5,096 \\
\hline Billing & 141,350 & 371,190 & 229,840 & - & 7,116 \\
\hline Nursing & 40,247 & 64,770 & 24,523 & - & 691 \\
\hline Ambulance & 28,112 & 234,660 & 206,548 & - & 30,828 \\
\hline Hospital & 159,480 & 163,200 & 3,720 & 83 & 63 \\
Departments & & & & & \\
\hline
\end{tabular}

their patients. Furthermore, although cost saving is an important aspect of benefits to the network, the quality and timeliness aspects of the connectivity are also important, and they are not included in the report of the cost savings.

The benefit of using WHIN was estimated based on the cost saving per transaction of $\$ 2.50$ for clinical transactions, and $\$ 1.00$ for referral requests. We reasoned that the savings in claims and payor eligibility have the same nature as referrals, and hence should have similar savings of $\$ 1.00$, and e-mails and CRS should have half the saving as referrals, and hence a cost-saving benefit of $\$ 0.50$ per transaction.

Based on the profiles, we estimated the cost and benefit of using WHIN for each user category over a five-year period, as reported in Table III.

The information user pays a one-time fee for connectivity, and then a monthly fee, which is partly based on their use of the network as well as on the number of physicians (if the node is of MD or clinic type), number of workstations, and LAN. The five-year cost for each user type was based on the fee structure of each user category.

Analysis: According to Table III, in the benchmark case, the constraint (25) in D4 is satisfied for all node categories, in that all nodes connected to the network benefit from it, but to a varying degree. Large clinics, billing services, and ambulance services reap the largest net benefit from connectivity. However, when we compute the net benefit per physician or per user, the picture changes. Net benefits per physician or user could indicate the efficiency of use in each type of node. Ambulance services are the most efficient users of the network. They have a relatively small number of users that use the system extensively, hence generating significant net benefits. Hospital departments, on the other hand, have a large number of staff physicians and users, but utilize the network the least; hence, are they the least efficient users of the network. Large clinics reap the largest net benefit from the network. However, their net benefits per physician and per user indicate that these clinics could substantially increase their benefit by more extensive utilization of the network services. The relatively low efficiency of the larger medical units indicates the opportunity for significant gain by redesigning and integrating their existing information systems with the network. 
Hospitals are the information providers, and they are the ones who benefit most from connectivity to various information users. In other words, when a hospital is connected to ten clinics, every time any one of these clinics makes a request for a clinical record, the system saves the hospital $\$ 5.10$ in cost alone, not considering the patient and physician satisfaction and quality of health service delivery. The fee structure of information providers is much higher both for the one-time fee and monthly fees, and depends partly on the number of staff physicians in the hospital. The details are not reported here for confidentiality reasons.

We set up D4 model using 11 nodes (ten information users and one large hospital), assuming that all ten node types are connected to this hospital type. As a benchmark for minimum benefits, we allowed benefits only between the hospital and the information users, and not among the information users.

This analysis led to the conclusion that connectivity to WHIN is highly cost-effective for all node types. For this analysis, we defined a fully dedicated equivalent node as a node whose transactions are exclusively with one hospital. The fully dedicated connectivity is not a common practice in most cases. However, a hospital may have four large clinics on WHIN, each of which has $25 \%$ of its transactions with that hospital. In that case, four large clinics would be equivalent to one fully dedicated large clinic.

Our analysis shows that a large hospital with even one fully dedicated equivalent node in each category stands to benefit substantially. For example, a large hospital would have a net benefit of about \$2.4 million in five years if one fully dedicated equivalent of each category is connected to it. For a large hospital to reach a break-even point of recovering its costs, it is enough to have only two large clinics, or alternatively, a large clinic plus one medium clinic (or large MD office) plus one billing service (all fully dedicated equivalent). The hospital reaches high profitability very quickly as the number of nodes in each category increases. This conclusion is not sensitive to the size of hospital if the nodes are fully dedicated. However, when the transactions are spread among the hospitals, it would take a larger number of nodes to have a fully dedicated equivalent node. Then a higher number of nodes would be needed to reach profitability. Hence, the profitability of the hospital is directly related to the number of nodes using its information as well as the percentage of the transactions of each node directed to that hospital.

The profitability of the network owner would be significant if it could contain its operating costs and the overhead for installing and maintaining the network. In our analysis, we did not have accurate figures for overhead and the variable (operating) costs of installing and maintaining each node. However, if the network can keep its installation and operating costs at $60 \%$ of what it charges, a network with only one node of each type would have a cash inflow of about $\$ 670000$ in $5 \mathrm{yr}$ (this figure excludes the start-up cost and administrative overhead). The same network would generate $\$ 3.5$ million in benefits for its participants, of which $\$ 2.4$ million belongs to the participating large hospital. The important element in this analysis is that the installation and operating costs of each node must be lower than the fee the network charges to the information provider and user. Otherwise, the network would not be profitable as new nodes are added to the network.
As the number of nodes in each category increases, so does the profit of the network owner-as well as the total benefits for the network participants. This result shows that HINs, based on the existing data, produce value for their owners and participants. Considering the fact that access to networks could improve the quality of care through fast access to accurate and up-to-date patient information, HINs should be one of the major strategic tools of health organizations. The organizations that pioneer this technology will have the potential to gain substantial competitive advantage.

\section{REDESIGNING THE WHIN ARCHITECTURE}

The above analysis was based on the existing structure of WHIN. The next question was to use our decision framework to redesign the WHIN architecture. In the new architecture, we remove the existing dichotomy of information providers and information users, and allow all nodes (including hospitals) to be both providers and users of information. We make no distinction between the hospital and its departments in that all departments of a hospital may use and provide information.

Such a redesign needs to resolve a number of issues. The implementation of this design, whether enterprise-oriented, consortium-based, or community-based, raises issues regarding the confidentiality of patient information and the security of the information system [45]. Among these issues of great concern are integrity, availability, source control, errors/omissions, and leakage of patient information [26], [32], [51]. Such concerns are easier to address in enterprise-owned networks than open systems. However, with the increasing value of information networks in health care, none of these challenges could pose an insurmountable barrier to building such networks.

When nodes are both the providers and users of information, it would be necessary to compute the pairwise benefit of connectivity for each node. We report this analysis for the clinical transactions and clinical node types only. As before, we use $\$ 5.10$ savings for the provider of information and $\$ 2.50$ for the user of information. In this analysis, a node could be both the provider of information (saving \$5.10) when other nodes access it and the user of information (saving \$2.50) when it accesses other nodes.

We report our estimation of the pairwise benefits of nodes over five years in Table IV. We included ten hospitals in the region that each node may potentially use. Each hospital has an equal chance of being used by a node. We use one type of hospital to simplify the analysis. We assume that there are 2000 MDs in the network's region. Each clinical inquiry may have the chance of one in 2000 to be directed at a given MD. The entries in Table IV show pairwise benefits between two single nodes of each type. As our D4 formulation shows, the benefit to a given node is the product of the pairwise benefit and the number of nodes in each category.

Using the WHIN data, we estimated that an MD generates 100 clinical queries to hospitals per month. We also made a conservative estimate of 100 transactions generated by each MD for information generated by other MDs (in other clinics or MD offices). A small clinic or MD office has to use the network to get information generated by other MDs. However, a medium or 
TABLE IV

Five-Year Pairwise Benefits (IN DOLlars) FOR ONE NODE IN EACH CATEGORY

\begin{tabular}{l|r|r|r|r|r|r|r}
\hline Category & $\begin{array}{c}\text { Small } \\
\text { Clinic }\end{array}$ & $\begin{array}{c}\text { Med. } \\
\text { Clinic }\end{array}$ & $\begin{array}{c}\text { Large } \\
\text { Clinic }\end{array}$ & $\begin{array}{c}\text { Small } \\
\text { MD }\end{array}$ & $\begin{array}{c}\text { Med. } \\
\text { MD }\end{array}$ & $\begin{array}{c}\text { Large } \\
\text { MD }\end{array}$ & Hospital \\
\hline Small-Clinic & 876 & 1,860 & 8,268 & 170 & 357 & 673 & 50,166 \\
\hline Med. Clinic & 1,648 & 3,402 & 14,778 & 319 & 652 & 1,203 & 78,354 \\
\hline Large Clinic & 6,575 & 13,184 & 55,860 & 1,273 & 2,528 & 4,549 & 136,674 \\
\hline Small MD & 170 & 360 & 1,600 & 33 & 69 & 130 & 11,769 \\
\hline Med. MD & 316 & 652 & 2,834 & 61 & 125 & 231 & 17,520 \\
\hline Large MD & 535 & 1,074 & 4,549 & 104 & 206 & 364 & 49,167 \\
\hline Hospital & 28,272 & 66,576 & 319,200 & 5,472 & 12,768 & 25,992 & 136,674 \\
\hline
\end{tabular}

large MD office or clinic would have less need to access information generated by other MDs outside its own office, because it is possible that some needed clinical information would be available in its own office. Hence, we applied a $70 \%$ and $50 \%$ hit ratio for network access to other MD-generated information for medium and large clinics and MD offices, respectively. This is a conservative estimate for a network with many interacting nodes.

We used the benefits in Table IV in the D4 model. We kept the cost and fee structures the same as before. That is, we used the existing fee structure for the nodes and an operating cost at the level of $60 \%$ of the charged fee. We have performed the analysis only for large hospitals.

We computed the results using D4 for four scenarios. In these scenarios, we fixed the maximum number of potential nodes of nonhospital types, and changed the number of hospitals from one to two, four, and six; and the maximum number of total nodes to be connected to the network from 50, to 100, 200, and 300 . We also computed the total profits (benefits - cost) generated to all the participants in the network. The summary of the scenarios is reported in Table V.

We assumed a start-up cost of $\$ 2$ million for building the network for a 50-node connection. This overhead was then increased to $\$ 3.0, \$ 3.5$, and $\$ 4.0$ million as the number of nodes increased from 50 to 100,200 , and 300 , respectively. We have assumed the economies of scale with respect to adding nodes to a 100-node network. That is, when the number of nodes increases by a factor of three (from 100 to 300), the requirements for additional hardware and software resources would change by a factor far less than three.

As Table V shows, clinics are preferred to MD offices at the start. As the connected clinics reach their maximum potential numbers, then MD offices are added to the network. Not all potential medium and small MD offices are added to the network in the four scenarios we have reported here. They have lower priority and may be connected only when the capacity of the network goes beyond 300 nodes. This low priority is consistent with the fact that some small and medium MD offices do not have the needed resources in computers and manpower in place to be able to connect to the network. They would need additional investment for such connectivity.

The profits of the network owner as well as the participants increase as the total number of nodes is increased. The profit of the network owner (excluding its fixed operating costs) in-
TABLE $\mathrm{V}$

SCENARIOS FOR REDESIGNING THE NETWORK

\begin{tabular}{l|r|r|r|r}
\hline \multirow{2}{*}{$\begin{array}{c}\text { Assumptions and Solutions of } \\
\text { the Scenario Analysis( }{ }^{*} \text { ) }\end{array}$} & \multicolumn{5}{|c}{ Scenario } \\
\cline { 2 - 5 } & \multicolumn{1}{c|}{$\mathbf{2}$} & \multicolumn{1}{c}{$\mathbf{3}$} & \multicolumn{1}{c}{$\mathbf{4}$} \\
\hline \# of Small Clinics: Max/Solution & $100 / 29$ & $100 / \mathbf{7 8}$ & $100 / \mathbf{1 0 0}$ & $100 / \mathbf{1 0 0}$ \\
\hline \# of Med. Clinics: Max/Solution & $50 / 0$ & $50 / \mathbf{0}$ & $50 / \mathbf{5 0}$ & $50 / \mathbf{5 0}$ \\
\hline \# of Large Clinics: Max/Solution & $20 / \mathbf{2 0}$ & $20 / \mathbf{2 0}$ & $20 / \mathbf{2 0}$ & $20 / \mathbf{2 0}$ \\
\hline \# of Small MDs: Max/Solution & $100 / 0$ & $100 / \mathbf{0}$ & $100 / \mathbf{0}$ & $100 / \mathbf{5 4}$ \\
\hline \# of Med. MDs: Max/Solution & $50 / \mathbf{0}$ & $50 / \mathbf{0}$ & $50 / \mathbf{6}$ & $50 / \mathbf{5 0}$ \\
\hline \# of Large MDs: Max/Solution & $20 / 0$ & $20 / \mathbf{0}$ & $20 / \mathbf{2 0}$ & $20 / \mathbf{2 0}$ \\
\hline \# of Hospitals: Max/Solution & $1 / \mathbf{1}$ & $2 / \mathbf{2}$ & $4 / \mathbf{4}$ & $6 / \mathbf{6}$ \\
\hline Maximum Number of Nodes & 50 & 100 & 200 & 300 \\
\hline Start-up Cost in Million \$ & 1.0 & 2.0 & 3.5 & 4.0 \\
\hline Network Profit in Million \$ & $\mathbf{2 . 2}$ & $\mathbf{4 . 1}$ & $\mathbf{8 . 2}$ & $\mathbf{9 . 5}$ \\
\hline Participants Profits in Million \$ & $\mathbf{8 . 1}$ & $\mathbf{1 0 . 5}$ & $\mathbf{1 7 . 3}$ & $\mathbf{1 9 . 4}$ \\
\hline
\end{tabular}

(*) Values in boldface represent the solution in each scenario.

creases from $\$ 2.2$ to $\$ 9.5$ million as the number of connected nodes increases from 50 to 300 . At the same time, the total net value-added for the participants in the network increases from \$8.1 million in the first scenario to \$19.4 million in the fourth scenario. The total value-added for the participants is more than twice the profit of the network owner.

Our analysis shows how to design HINs and perform cost-benefit analysis for them using our decision support systems. Our approach could bridge the gap among the diverse groups of decision makers involved with health information systems. Furthermore, the results of our data analysis indicate that HINs, at least in the case of WHIN, could provide substantial value to network owners as well as to information users and providers.

The idea of network for service delivery is not unique in health industry. Similar networks could be used for providing services on other industries. Leung et al. [33] report on a framework for a global community logistics network for the air cargo industry in Hong Kong. The members of this community consist of freight forwarders, integrators, warehousing and distributing agents and service providers, airlines, airport authorities, and cargo terminal operators. Our models could be useful in analyzing the design of such network in other industries as well. Furthermore, Borriello and Want [3], in their review of upcoming web-based network technologies, predict that, alongside home automation, health monitoring through the web access will be an important application of web-based technologies. Such development will require secure web-based architectures and extend the active health network participants to include patients who are attached to web-based health monitoring devices. Our framework could be applied to cost-benefit analysis of designing such systems and for prioritizing the connectivity of various type patients to such systems.

\section{CONCLUSION}

The health care industry is going through a period of radical changes in its market structure and in the internal organization 
of its member companies. In this period, the effective utilization of technology plays a strategic role. HINs are an important technological tool that could facilitate these changes. While the importance of such systems is well recognized in the industry, government, and academia, there is no formal framework for designing and performing cost-benefit analysis for such networks. In this research, we provided such a framework and use it for analyzing and redesigning one of the pioneering systems in this area.

In this paper, we formulated a number of decision models for designing an HIN with different decision focuses, and performing cost and benefit analysis on a formal and rigorous basis. The process of our empirical investigations led us to formulate four decision models for determining the connectivity of candidate nodes to a network for exchanging patient information. The first model determines the design of a new network by maximizing the owner's profit and ensuring benefits for all nodes connected to the network. The second decision model identifies the minimum number of nodes needed to make a network break even. The third model determines the expansion of an existing network. The fourth model performs similar analysis for categories of nodes.

We applied our framework to an existing health network: WHIN, a pioneering enterprise in its own right. The existing architecture of this network is that hospitals are the providers of information to the network, and MD offices, clinics, and other health organizations are the users of the information.

We categorized WHINs information users into ten user-node types, and three providers of information (three hospital types). We collected data from WHIN regarding the users of the network and its fee structures. A user profile for each node type was developed. We also computed the average number of monthly transactions from WHINs records. This made us able to estimate the users' costs and charged fees. Since there was a prior study regarding the cost-saving aspect of providing and using the network, we were able to estimate the benefits enjoyed by each category from connectivity to the network.

We were surprised by the extent and variability of the average net benefits that the node types incurred from using the network and providing information to it. The hospitals, which are the major providers of information, stand to benefit the most from it. Medium-size clinics, billing services, and ambulance services also benefit substantially from connectivity to the existing network.

The information users do not utilize the network with the same efficiency. Some larger medical units and hospital departments are not taking full advantage of the system, and have the potential for significant gains if they integrate the network with their internal information systems and re-engineer their process to increase their use of network services. The profit of the network owner depends on its cost structure for installing and maintaining each node type. If the owner succeeds in keeping costs to about $60 \%$ of the charged fees, it stands to reap substantial benefits.

We estimated pairwise benefits from the data provided by WHIN and certain assumptions regarding the number of MDs and hospitals in the region. Our analysis showed that the nodes would be of great benefit to both the network owner and net- work participants. The network owner stands to reap substantial benefits as long as two conditions are satisfied.

1) The network should be fully utilized with the transactions by each node type reaching the average level for that type.

2) The network owner should have the ability to contain its installation and maintenance costs.

We believe that our framework provides a rigorous basis for planning and performing cost-benefit analysis for information networks. It quantifies the overall design of such networks, and hence makes it possible to decide connectivity allocation to potential nodes. We were able to utilize our approach in analyzing the existing structure of WHIN and in redesigning its architecture. Our analysis of WHIN showed that indeed such networks provide substantial values to all parties involved-network owners as well as information users and providers.

This work could be extended by applying the proposed models in evaluating other HINs as well as in assessing networks designed for interorganizational cooperation in other industries. It could also be used for prioritizing access and use in the settings where the use of the network may require substantial commitment of financial and human resources.

\section{ACKNOWLEDGMENT}

The authors would like to thank K. Lassila who brought the existence of the problem addressed in this paper to the attention of one of the authors.

\section{REFERENCES}

[1] J. G. Anderson, "Clearing the way for physicians' use of clinical information systems," Commun. ACM, vol. 40, no. 8, pp. 83-90, Aug. 1997.

[2] E. L. Barker, A. Friede, and A. D. Moulton, "CDC's information network for public health officials (INPHO): A framework for integrated public health information and practice," J. Public Health Manage. Practice, vol. 1, pp. 43-47, Jan. 1995.

[3] G. Borriello and R. Want, "Embedded computation meets the world wide web," Commun. ACM, vol. 43, no. 5, pp. 59-66, May 2000.

[4] C. P. Brennan, "Managed care and health information networks," $J$. Health Care Finance, vol. 21, no. 4, pp. 1-5, Summer 1995.

[5] P. F. Brennan, S. J. Schneider, and E. Tornquist, Eds., Information Networks for Community Health. New York: Springer-Verlag, 1997.

[6] D. Braly, "Telecom use grows for regional data access," Health Manage. Technol., vol. 16, no. 6, pp. 22-24, June 1995.

[7] K. M. Bretthauer and M. J. Côté, "A model for planning resource requirements in health care organizations," Decision Sci., vol. 29, no. 1, pp. 243-270, Winter 1998.

[8] M. Brown, "Mergers, networking, and vertical integration: Managed care and investor-owned hospitals," Health Care Manage. Rev., vol. 21, no. 1, pp. 29-37, Winter 1996.

[9] N. J. Brownstein et al., "CHINs: A public health perspective," in Information Networks for Community Health, P. F. Brennan, S. J. Schneider, and E. Tornquist, Eds. New York: Springer-Verlag, 1997, pp. 117-134.

[10] R. L. Bushshur, J. H. Sanders, and G. W. Shannon, Eds., Telemedicine: Theory and Practice. Springfield, IL: Charles Thomas, 1997.

[11] D. Coddington, "Information systems and integrated healthcare: An essential partnership," Health Manage. Technol., vol. 16, no. 7, pp. 38-40, July 1995.

[12] J. Darnell, "LIS technologies must grow into the 21st century," Computers in Healthcare, vol. 14, no. 9, pp. 41-42, Sept. 1993.

[13] L. Delesie, "Bridging the gap between clinicians and health managers," Eur. J. Oper. Res., vol. 105, no. 2, pp. 248-256, Mar. 1998.

[14] W. M. Detmer and E. H. Shortliffe, "Using the Internet to improve knowledge diffusion in medicine," Сотmиn. ACM, vol. 40, no. 8, pp. 101-108, Aug. 1997.

[15] A. Dowling, "CHINS - The current state," in Information Networks for Community Health, P. F. Brennan, S. J. Schneider, and E. Tornquist, Eds. New York: Springer-Verlag, 1997, pp. 15-40. 
[16] C. Dunbar, "University of Iowa links rural providers," Health Manage. Technol., vol. 15, no. 8, pp. 20-26, Aug. 1994.

[17] _ , "The networking standards evolution: Toward a real electronic medical record," Comput. Healthcare, vol. 11, no. 2, pp. 18-21, Feb. 1990.

[18] B. A. Friedman and W. Mitchell, "Community health information networks (CHINS) and their relationship to telemedicine," in Telemedicine: Theory and Practice, R. L. Bushshur, J. H. Sanders, and G. W. Shannon, Eds. Springfield, IL: Charles Thomas, 1997, pp. 53-76.

[19] T. W. Ferratt et al., "Surmounting health information network barriers: The greater Dayton area experience," Health Care Manage. Rev., vol. 23, no. 1, pp. 70-76, Jan. 1998.

[20] F. M. Furukawa, "CHINs in progress: Evolving to the next level," in CHIN 100 Market Directory: ComNet Soc., 1996.

[21] D. Garets and D. Hanna, "Emerging managed care technologies," Health Manage. Technol., vol. 19, no. 11, pp. 28-32, Nov. 1998.

[22] M. Hagland, "Glimpses of a Web-enabled future," Health Manage. Technol., vol. 19, no. 4, pp. 22-29, Apr. 1998.

[23] R. Hard, "Brigham and women's teams PACS, RIS technologies," Health Manage. Technol., vol. 15, no. 3, pp. 16-20, Mar. 1994.

[24] Information Infrastructure Task Force, The National Information Infrastructure. Washington, DC: The White House, Sept. 15, 1993.

[25] M. G. Kahn, "Enterprise-wide clinical data integration," in Information Networks for Community Health, P. F. Brennan, S. J. Schneider, and E. Tornquist, Eds. New York: Springer-Verlag, 1997, pp. 41-54.

[26] M. E. Kabay, "Information systems security and management in today's healthcare environment," presented at the 15th Annu. Assoc. Appl. Clinical Inform. Syst. Conf., Snowbird, UT, Mar. 1995.

[27] D. G. Kilman and D. W. Forslund, "A international collaboratory based on virtual patient records," Commun. ACM, vol. 40, no. 8, pp. 111-117, Aug. 1997.

[28] K. W. Kimel, "The rural televillage: Creating a new strategy for rural development," in White Paper presented at the Creating Televillage Conf., Tampa, FL, 1994.

[29] A. Kumar and J. G. Motwani, "Management of health care technology literature (1979-1997): A multidimensional introspection," IEEE Trans. Eng. Manage., vol. 46, pp. 247-264, Aug. 1999.

[30] K. S. Lassila and R. H. Cheng, "Taming the RHINO: Interorganizational implementation issues of regional health information networks," in Proc. Inaugural America's Conf. Inform. Syst., Aug. 1995, pp. 25-27.

[31] K. S. Lassila et al., "Assessing the impact of community health information networks: A multisite field study of the Wisconsin Health Information Network," Top Health Inform. Manage., vol. 18, no. 2, pp. 64-76, 1997.

[32] L. M. Lawrence, "Safeguarding the confidentiality of automated medica information," J. Quality Improvement, vol. 20, no. 11, pp. 639-645, Nov. 1994

[33] L. C. Leung, W. Cheung, and Y. V. Hui, "A framework for logistics e-commerce community network: The Hong Kong cargo air industry," IEEE Trans. Syst., Man, Cybern. A, vol. 30, pp. 446-455, July 2000.

[34] J. Morrissey, "Info systems survey," Modern Healthcare, vol. 25, no. 7 , pp. 65-72, Feb. 1995

[35] — , "Full speed ahead," Modern Healthcare, vol. 26, no. 10, pp 97-108, Mar. 1996.

[36] — , "Partners THINC big," Modern Healthcare, vol. 27, no. 14, p. 80, Apr. 1997.

[37] — "Integration sacrificed for Y2K preparation," Modern Healthcare, vol. 29, no. 18, pp. 31-34, May 1999.

[38] J. Nolan, "Networked labs build continuity of care," Health Manage. Technol., vol. 16, no. 3, pp. 14-19, Mar. 1995.

[39] D. Nutkis and R. Golob, "Community health information networks," in Pulse: Ernst \& Young LLP, Spring 1995, pp. 11-15.

[40] K. R. Pemble, "Information infrastructure for health communities: The Wisconsin health information network," in Information Networks for Community Health, P. F. Brennan, S. J. Schneider, and E. Tornquist, Eds. New York: Springer-Verlag, 1997, pp. 73-99.

[41] C. Payton and P. F. Brennan, "How a community health information network is really used," Commun. ACM, vol. 42 , no. 12 , pp. 83-89, Dec. 1999.

[42] D. S. Puskin, "Opportunities and challenges to telemedicine in rural America," J. Med. Syst., vol. 19, no. 1, p. 59, Jan. 1995.

[43] D. S. Puskin, C. L. Mintzer, and C. J. Wasem, "Telemedicine: Building rural systems for today and tomorrow," in Information Networks for Community Health, P. F. Brennan, S. J. Schneider, and E. Tornquist, Eds. New York: Springer-Verlag, 1997, pp. 271-286.
[44] W. Raghupathi, "Health care information systems," Commun. ACM, vol. 40, no. 8, pp. 81-82, Aug. 1997.

[45] T. C. Rindfleisch, "Privacy, information technology, and health care," Commun. ACM, vol. 40, no. 8, pp. 93-100, Aug. 1997.

[46] R. D. Rubin and M. C. Aukema, "CHMIS Evolution: The metamorphosis of community health information movement," in Information Networks for Community Health, P. F. Brennan, S. J. Schneider, and E. Tornquist, Eds. New York, NY: Springer-Verlag, 1997, pp. 101-115.

[47] O. Sheng et al., "Organizational management of telemedicine technology: Conquering time and space boundaries in health care industry," IEEE Trans. Eng. Manage., vol. 46, pp. 265-278, Aug. 1999.

[48] C. H. Tuohy, "Dynamics of a changing health sphere: The United States, Britain, and Canada," Health Affairs, vol. 18, no. 3, pp. 114-134, May/June 1999

[49] - Accidental Logic: Dynamics of Change in the Health Care Arena in The United States, Britain, and Canada. New York: Oxford Univ. Press, 1999.

[50] R. Ziegler, Ed., Change Drivers: Information Systems for Managed Care. Chicago, IL: Amer. Hospital Assoc., 1998.

[51] U.S. Congress, Office of Technology Assessment, "Protecting privacy in computerized medical information," U.S. Govt. Printing Office, Washington, DC, OTA-TCT-576, 1993.

[52] U.S. Congress, Office of Technology Assessment, "Rural America at the crossroads: Networking for the future," U.S. Govt. Printing Office, Washington, DC, OTA-TCT-471, April 1991

[53] Wisconsin Health Information Network (WHIN) 2000 [Online]. Available: http://www.whin.net.

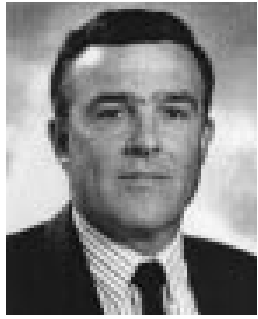

Oded Berman received the Ph.D. degree in operations research from the Massachusetts Institute of Technology, Cambridge, in 1978.

He has been with the Electronic Systems Laboratory at MIT and the University of Calgary, Calgary, $\mathrm{AB}$, Canada. He was also the Chairman of the Department of Management Sciences at the University of Massachusetts, Amherst. Currently, he is a Full Professor and the former Associate Dean of Programs at the Joseph L. Rotman School of Management at the University of Toronto, Toronto, ON, Canada. He has published over 110 articles and has contributed to several books in his field. His main research interests include operations management in the service industry, location theory, network models, and software reliability. He is an Associate Editor for Operations Research, Management Science, Transportation Science, and a Member of the Editorial Boards for Computers and Operations Research and the Journal of Service Research.

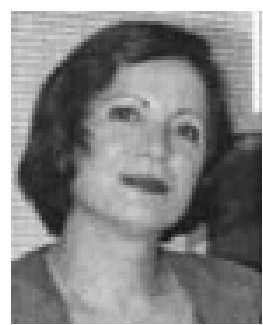

Fatemeh "Mariam" Zahedi received the Ph.D. degree from Indiana University, Bloomington, in 1975.

Currently, she is a Wisconsin Distinguished Professor, MIS Area at the School of Business, University of Wisconsin, Milwaukee. Her present areas of research include IS quality and satisfaction, e-commerce and web development, intelligent decision support systems, and IS-related policies, and decision analysis. She has published extensively in refereed journals including MIS Quarterly, Decision Sciences, IEEE TRANSACTIONS ON SOFTWARE ENGINEERING, IIE Transactions, European Journal of Operations Research, Operations Research, Computers and Operations Research, Interfaces, International Journal of Quality and Reliability Management, Journal of Information Technology Management, Journal of Review of Economics and Statistics, and Empirical Economics: Socio-Economic Planning Sciences. She is the author of two books: Quality Information Systems (New York: Fraser, 1995) and Intelligent Systems for Business: Expert Systems with Neural Network, (London, U.K.: Wadsworth, 1993) and serves on the editorial board of a number of journals. She has also contributed on the topic of information systems to International Encyclopedia of Business and Management, group decisions in Encyclopedia of Management Science and Operations Research and quality control in Wiley Encyclopedia of Electrical and Electronic Engineering. 


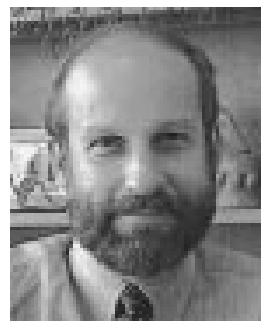

Kim R. Pemble received the M.S. degree in biometry and health information systems from the University of Minnesota, Minneapolis, in 1982.

He is currently Assistant Professor in the Healthcare Administration and Informatics Program, School of Allied Health Professions, University of Wisconsin, Milwaukee, and Vice President of Information Technology for Infinity HealthCare, Inc., Mequon, WI. His 20 years of experience in healthcare informatics includes serving as Director of Technical Operations and Vice President of Operations and Development for the Wisconsin Health Information Network (WHIN) from 1993 to 1997. His research interests include regional health networks and data repositories, as well as the application of geographic information systems in healthcare business and clinical decision-making. He has authored and coauthored several papers and presentations on topics including spatially enabling the CPR and the role of health networks and regional data repositories. 